\title{
可见光诱导简单烷烃碳氢活化以实现菲啶类化合物的绿色合成
}

\author{
李文艺 $a$ 何卫民*,b \\ ( ${ }^{a}$ 衡阳师范学院化学与材料科学学院 湖南衡阳 421010) \\ ( $b$ 南华大学化学化工学院 湖南衡阳 411201)
}

\section{Visible Light-Induced Green Synthesis of Phenanthridines via C-H Activation of Simple Alkanes}

\author{
Li, Wenyi ${ }^{a} \quad \mathrm{He}$, Weimin $^{*, b}$ \\ ( ${ }^{a}$ College of Chemistry and Materials Science, Hengyang Normal University, Hengyang, Hunan 421008) \\ ( ${ }^{b}$ School of Chemistry and Chemical Engineering, University of South China, Hengyang, Hunan 421001)
}

简单烷烃作为石油和天然气的主要成分, 广泛存在 于自然界中. 但由于烷烃碳氢活化过程中本身的化学惰 性和难以控制的化学选择性, 对烷烃的利用仍以燃烧供 能为主, 如何将此类丰富的有机碳源直接作为合成子进 行高原子利用率及高附加值转化, 是一个函待解决的科 学问题. 有机光化学合成作为一种条件温和、环境友好 且可持续性的合成策略, 受到了化学工作者的广泛关 注. 其中, 光诱导氢原子转移(HAT)过程为碳氢活化领 域提供了一类温和、高效、可行的合成方案, 与传统热 反应相比, 光反应过程大大避免了能源浪费与环境污染 问题. 近年来, 光诱导碳氢活化领域取得了迅猛发展, 大量具有代表性的研究工作相继被报道 ${ }^{[1]}$.

氮杂环类化合物广泛存在于天然产物和生物活性 分子中, 其出色的成盐及促溶能力, 使其在药物分子开 发方面发挥着重要作用 ${ }^{[2]}$. 菲啶及其衍生物作为一类高 价值药物活性分子, 表现出丰富的抗癌、消毒和杀菌等 活性. 碳氢活化的异腈与自由基加成环化反应是一类行 之有效的菲啶合成方法, 但此类反应所需温度通常较 高, 且需要一些强氧化剂共同参与反应, 如有机过氧化 物、氟试剂以及高价碘试剂等, 因此该类反应面临成本 较高、副产物多、原子利用率低及官能团耐受性差等问 题. 鉴于此, 开发一种绿色环保、经济高效的基于碳氢 活化合成菲啶类化合物的方法十分必要.

近日, 大连理工大学张大显学院金云鹤/段春迎课 题组 ${ }^{[3]}$ 在其长期对光催化合成反应的研究基础上, 开发 了一种在无金属条件下, 以 2-异氧基联苯和简单烷烃为
底物, 2,4,5,6-四(9-咔唑基)-间苯二腈(4-CzIPN)为光敏 剂, 双氧水溶液作为氢原子转移催化剂前驱体, 有效实 现了基于烷烃碳氢活化的菲啶衍生物绿色合成新方 法 $^{[4]}$, 相比于传统方法取得了明显进步(Scheme 1).

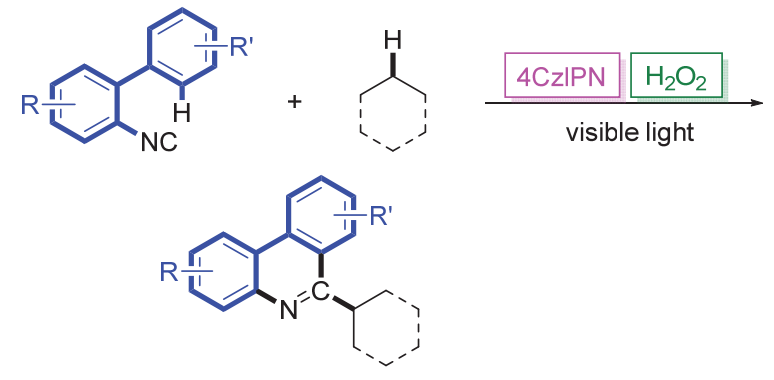

32 examples, up to $94 \%$ yield

图式 1 光诱导烷烃 $\mathrm{C}-\mathrm{H}$ 键活化构建菲啶衍生物 Scheme 2 Photo-induced construction of phenanthridine derivatives via $\mathrm{C}-\mathrm{H}$ activation of alkanes

金云鹤/段春迎课题组以 2-异氧基联苯和环已烷作 为模板底物, 对反应所需的光敏剂、氧化剂、添加剂以 及溶剂进行了系统篎选. 研究发现在氮气及 $395 \mathrm{~nm}$ LED 光照的环境下, 以 4-CZIPN 作为光敏剂, $\mathrm{H}_{2} \mathrm{O}_{2}$ 作为 氧化剂, 乙腈作为溶剂以及 $N, N$-二异丙基乙胺(DIPEA) 作为添加剂, 能够以 $89 \%$ 的产率得到目标产物 6-环已基 菲啶. 对照实验表明, 光照和惰性气体氛围是反应进行 的必要条件. 随后, 该课题组考察了异腈和烷烃的底物 适应性(Scheme 2). 对于 2-异氰基联苯, 研究发现分别 在两个苯环上引入不同的取代基后, 都可以以较高收率

* Corresponding author. E-mail: weiminhe2016@yeah.net. Published online October 15, 2021. 
得到相应的目标产物, 说明反应体系对不同官能团的耐 受性良好. 值得一提的是, 三球啶(trisphaeridine)作为一 种非常有价值的生物碱, 其烷基化衍生物同样能够通过 该策略成功合成. 对于烷烃底物, 环烷和链状烷烃都具 有较好的适用性, 两种气态烷烃丁烷和异丁烷同样适用 于该反应体系. 区域选择性研究表明, 该碳氢活化过程
选择性发生在仲碳和叔碳 $\mathrm{C}-\mathrm{H}$ 键上, 而伯碳 $\mathrm{C}-\mathrm{H}$ 键 不会参与反应. 为进一步拓展应用范围, 该课题组还成 功实现了对醚类、胺类和醛类等不同类别有机化合物的 $\mathrm{C}-\mathrm{H}$ 活化，合成了对应的菲啶衍生物. 最后, 为了验证 这个方法的实用性，作者还对模板反应进行了克量级放 大实验，发现反应收率依旧可观.

\section{2-Isocyanobiphenyl scope}

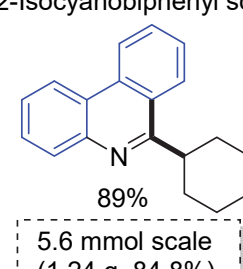<smiles>Cc1ccc2c(c1)c(C1CCCCC1)nc1ccccc12</smiles><smiles>COc1ccc2c(c1)c(C1CCCCC1)nc1ccccc12</smiles><smiles>FC(F)(F)c1ccc2c(c1)nc(C1CCCCC1)c1ccccc12</smiles><smiles>COc1ccc2nc(C3CCCCC3)c3ccccc3c2c1</smiles><smiles>Clc1ccc2nc(C3CCCCC3)c3ccccc3c2c1</smiles>
$(1.24 \mathrm{~g}, 84.8 \%)$

$87 \%$

$83 \%$

$90 \%$

$87 \%$

$78 \%$<smiles>Cc1ccc2c(c1)nc(C1CCCCC1)c1ccccc12</smiles>

$80 \%$<smiles>c1ccc2c(c1)ccc1c3ccccc3nc(C3CCCCC3)c21</smiles>

$61 \%$<smiles>COc1ccc2c(c1)nc(C1CCCCC1)c1ccccc12</smiles>

$90 \%$<smiles>Fc1ccc2c(c1)c(C1CCCCC1)nc1ccccc12</smiles>

$82 \%$<smiles>Clc1ccc2c(c1)c(C1CCCCC1)nc1ccccc12</smiles>

$85 \%$<smiles>CC(C)(C)c1ccc2c(c1)c(C1CCCCC1)nc1ccccc12</smiles>

$73 \%$<smiles>Fc1ccc2c(c1)nc(C1CCCCC1)c1ccccc12</smiles>

$77 \%$<smiles>FC(F)(F)c1ccc2c(c1)nc(C1CCCCC1)c1ccccc12</smiles><smiles>COc1ccc2nc(C3CCCCC3)c3ccccc3c2c1</smiles>

$81 \%$

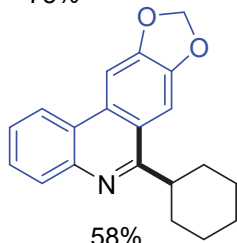

trisphaeridine derivative

Alkane scope

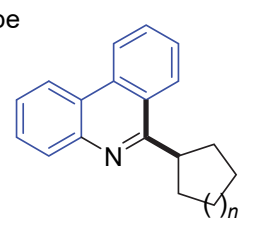

$n=1,87 \% ; n=3,82 \%$ $n=4,85 \% ; n=8,52 \%$<smiles>CC=C(CC)c1nc2ccccc2c2ccccc12</smiles>

$66 \%$, r.r. $1: 2=1: 1.8$

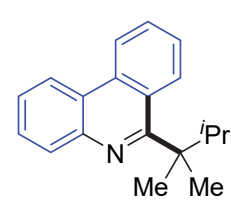

$81 \%$<smiles>COC(=[Pt])c1nc2ccccc2c2ccccc12</smiles>

$67 \%$, r.r. $1: 2=1.1: 1$<smiles>CC(C)Cc1nc2ccccc2c2ccccc12</smiles>

$58 \%$<smiles>CCC(C)(C)c1nc2ccccc2c2ccccc12</smiles>

$73 \%$, r.r. $1: 2=1.8: 1$

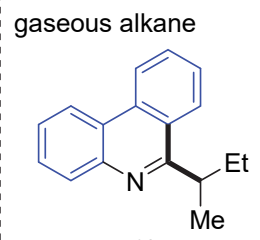

$45 \%$<smiles>CC(C)c1nc2ccccc2c2ccccc12</smiles>

$52 \%$

Other compounds<smiles>c1ccc2c(c1)nc(C1CCCO1)c1ccccc12</smiles><smiles>O=C(c1ccccc1)c1nc2ccccc2c2ccccc12</smiles>

$67 \%$<smiles>c1ccc2c(c1)nc(C1COCCO1)c1ccccc12</smiles>

$88 \%$<smiles>CCOC(C)c1nc2ccccc2c2ccccc12</smiles>

$94 \%$<smiles>CC(=O)N1CCCC1c1nc2ccccc2c2ccccc12</smiles>

$79 \%$<smiles>O=C(c1ccc(Cl)cc1)c1nc2ccccc2c2ccccc12</smiles>

$59 \%$<smiles>CN(C)C(=O)c1nc2ccccc2c2ccccc12</smiles>

$42 \%$

图式 2 底物拓展

Scheme 2 Substrate scope 
为了推测该体系的反应机理, 作者进行了包括自由 基捕获实验、动力学同位素实验、苂光淬灭实验以及量 子产率测定在内的一系列探究实验, 并阐明了如下机理 (Scheme 3): 首先, 在光照射下, 光敏剂 4-CzIPN 转变为 激发态 I, DIPEA 和 I 之间发生单电子转移(SET)得到自 由基 II 和 III, II 与双氧水间的 SET 进一步生成氢氧根 负离子和 HAT 活化试剂羟基自由基, 随后羟基自由基 和烷烃之间的 HAT 过程得到烷基自由基 IV, IV 被异腈 捕获后发生分子内加成反应分别得到自由基中间体 $\mathbf{V}$ 和 VI, VI 与 III 以及氢氧根负离子发生脱氢氧化便得到

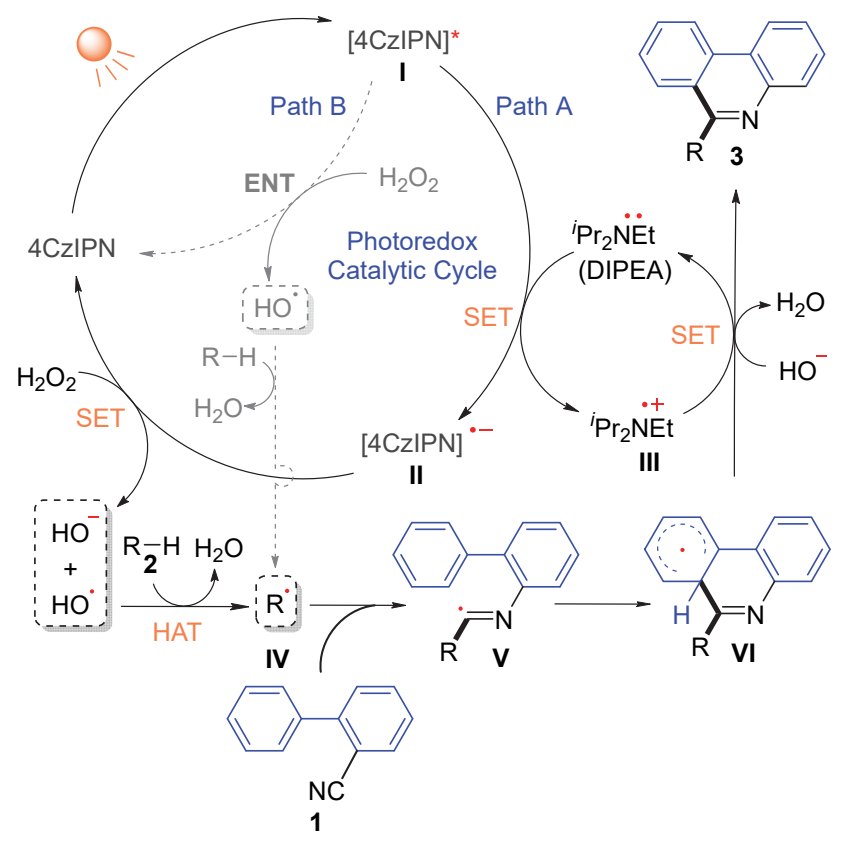

图式 3 反应机理

Scheme 3 Reaction mechanism
最终产物 3. 探究还发现, 当 DIPEA 不存在的时候, 反 应能够以中等产率得到目标产物, 这主要得益于 $\mathbf{I}$ 与双 氧水之间进行的能量转移过程, 因此添加剂 DIPEA 的 引入能够有效调控该体系的反应进程.

综上所述，金云鹤/段春迎课题组利用光诱导无金 属条件下的简单烷烃 $\mathrm{C}-\mathrm{H}$ 活化过程, 开发出一种条件 温和、绿色高效的菲啶类化合物制备方法. 该反应体系 具有原子利用率高、经济环保、底物和官能团适用性广 以及区域选择性高等优点. 该项策略对菲啶衍生物的合 成研究具有重要的指导意义.

\section{References}

[1] (a) Kong, Y.; Kim, J. K.; Li, Y.; Zhang, J.; Huang, M.; Wu, Y. Green Chem. 2021, 23, 1274.

(b) Pan, C.; Liu, P.; Wu, A.; Li, M.; Wen, L.; Guo, W. Chin. J. Org. Chem. 2020, 40, 2855.

(c) Li, M.; Wang, R.; Hao, W.; Jiang, B. Chin. J. Org. Chem. 2020, 40, 1540 (in Chinese).

(李梦帆，王榕，郝文娟，姜波，有机化学，2020,40,1540.)

[2] (a) Sun, K.; Xiao, F.; Yu, B.; He, W.-M. Chin. J. Catal. 2021, 42, 1921.

(b) Tang, H.; Pan, Y. Chin. J. Org. Chem. 2021, 41, 435 (in Chinese).

(唐海涛, 潘英明, 有机化学, 2021, 41, 435.)

(c) Han, Y.; Cui, X. Chin. J. Org. Chem. 2021, 41, 2929 (in Chinese).

(韩宇轩, 崔秀灵, 有机化学, 2021, 41, 2929.)

[3] (a) Zhang, T.; Jin, Y.; Shi, Y.; Li, M.; Li, J.; Duan, C. Coord. Chem. Rev. 2019, 380, 201.

(b) Jin, Y.; Zhang, Q.; Wang, L.; Wang, X.; Meng, C.; Duan, C. Green Chem. 2021, 23, 6984.

[4] Zhang, Y.; Jin, Y.; Wang, L.; Zhang, Q.; Meng, C.; Duan, C. Green Chem. 2021, 23, 6926. 